\title{
COVID-19 Vaccines: Lets Go for It
}

SARS CoV-2, also christened as COVID-19 is an acute respiratory illness caused by a novel coronavirus which originated from Wuhan China in 2019 and within a short span affected virtually each and every country around the globe, throwing healthcare preparedness and economy out of gear. The world was faced with a pandemic leading to overcrowded hospitals and overflowing mortuaries. Lockdowns imposed in countries jeopardized routine life never to normalize again. India has been among the top three countries with the maximum number of afflicted people. Thankfully, our statistics with respect to mortality is better as compared to the western world. The last year 2020 was completely COVIDISED if we may say so. The world economy is in doldrums and the health infrastructure is overstretched and fatigued. Everytime we feel that the disease is under control, the virus cleverly mutates to become more infective and develops increased ability to escape the immune surveillance.

Efforts to develop a vaccine against this scourge were initiated at a war footing across the globe. A number of vaccines were approved for emergency use since the scientific committee didn't have the time to wait further.

In India, two vaccines- Covaxin and Covishield have been approved by the Government of India for mass vaccination. The mammoth program was initiated with first preference for the healthcare workers and frontline warriors. In the next phases, elderly population followed by the rest of the population in a phased manner was planned. It is really incredible and praiseworthy about the immaculate planning and implementation of the initiative in our country where the diversity is both a boon as well as bane.

COVAXIN (BBV152), India's indigenous COVID-19 vaccine has been developed by Bharat Biotech in collaboration with the Indian Council of Medical Research (ICMR) - National Institute of Virology (NIV). This indigenous, inactivated vaccine is developed and manufactured in Bharat Biotech's BSL-3 (Bio-Safety Level 3) high containment facility. The vaccine is developed using Whole-Virion Inactivated Vero Cell derived platform technology and is an inactivated vaccine.

Covishield vaccine has been developed by Serum Insititute of India in collaboration AstraZe are ChAdOx1 nCoV- 19 Corona Virus Vaccines (Recombinant). Oxford The ChAdOx1 nCoV-19 vaccine (AZD1222) was developed at Oxford University and consists of a replication-deficient chimpanzee adenoviral vector ChAdOx1, containing the SARS-CoV-2 structural surface glycoprotein antigen (spike protein; nCoV-19) gene.

The other vaccines in the pipeline as stated in the ICMR portal include ZyCoV-D-Zydus Cadila announced that its plasmid DNA vaccine to prevent COVID-19, ZyCoV-D. Safety in Phase I clinical trial of ZyCoV-D in healthy subjects established as endorsed by the independent Data Safety Monitoring Board (DSMB). Zydus commenced Phase II trial.

Sputinik- Dr Reddys Laboratories Limited and Sputnik LLC are jointly conducting Multi-center, phase II/III adaptive clinical trial to assess safety and immunogenicity of Gam-COVID-Vac combined vector vaccine. The DGCl approved has been obtained for its use in India.

Biological E's novel Covid-19 vaccine -Biological E. Limited is conducting a prospective open label randomised Phase-I seamlessly followed by Phase-Il study to assess the safety, reactogenicity and immunogenicity of Biological E's novel Covid-19 vaccine containing Receptor Binding Domain of SARS-CoV-2 for protection against Covid-19 disease when administered intramuscularly in a two dose schedule $(0,28 \mathrm{D})$ to healthy volunteers.

BBV154 - Intranasal vaccine -Bharat Biotech is conducting Multicenter Study of an Intranasal Adenoviral vector COVID-19 vaccine (BBV154) in Healthy Volunteers. BBV154 is an intranasal vaccine stimulates a broad immune response - neutralizing lgG, mucosal lgA, and $T$ cell responses. It is undergoing Phase I trial.

COVOVAX-Indian Council of Medical Research and Serum Institute of India are jointly performing phase 2/3, observer-blind, randomized, controlled study to determine the safety and immunogenicity of COVOVAX [SARS-CoV-2 recombinant spike protein nanoparticle vaccine (SARS-CoV-2 rS) with Matrix-M1 ${ }^{\mathrm{TM}}$ adjuvant] in Indian adults.

There was a lot of speculation, miscommunication about the safety and efficacy of the vaccines. The intensive IEC campaign and word of mouth publicity allayed the fear of the population to a great extent and people are now more receptive and confident about the immunization programme. Let us all encourage 10 other people in our neighbourhood for vaccination. In this way we would be able to build a strong herd immunity and defeat this virus.

Dr Binita Goswami

Professor, Department of Biochemistry Maulana Azad Medical College, Delhi-110002 